\title{
Open Principle for Large High-Resolution Solar Telescopes
}

\author{
Robert H. Hammerschlag · Felix C. M. Bettonvil · Aswin P. L. Jägers • \\ Guus Sliepen
}

Received: 11 January 2008/Accepted: 11 July 2008/Published online: 24 December 2008

(C) The Author(s) 2008. This article is published with open access at Springerlink.com

\begin{abstract}
Vacuum solar telescopes solve the problem of image deterioration inside the telescope due to refractive index fluctuations of the air heated by the solar light. However, such telescopes have a practical diameter limit somewhat over $1 \mathrm{~m}$. The Dutch Open Telescope (DOT) was the pioneering demonstrator of the open-telescope technology without need of vacuum, now pursued in the German GREGOR. Important ingredients for this technology are primary beam completely open to natural wind flow, stiff but still open design by principal stiff overall geometries in combination with carefully designed joints and completely open-foldable dome constructions based on tensioned strong cloth. Further developments to large sizes are made within the framework of the design study for a European Solar Telescope (EST).
\end{abstract}

Keywords Solar telescopes · Open-telescope principle · Wind flushing ·

Stiff constructions · High spatial resolution - Seeing · Open towers · Open-foldable domes

\section{Introduction}

During solar observations, the telescope and its surroundings e.g. ground, building and dome are being heated by the solar light. Evacuated telescopes solve the problem for the optical beam inside the telescope. The R. B. Dunn Solar telescope in New Mexico with a 76-cm aperture was the first telescope of this type (Dunn 1964, 1969) and still operates successfully. The largest and sharpest vacuum telescope is the Swedish Solar Telescope

R. H. Hammerschlag ( $₫)$ · F. C. M. Bettonvil · A. P. L. Jägers · G. Sliepen

Astronomical Institute, Utrecht University, Princetonplein 5, 3584CC Utrecht, The Netherlands e-mail: r.h.hammerschlag@uu.nl

F. C. M. Bettonvil

Netherlands Foundation for Research in Astronomy (ASTRON), P.O. Box 2, 7990AA Dwingeloo, The Netherlands

A. P. L. Jägers · G. Sliepen

Technology Foundation STW, Utrecht, The Netherlands 
(SST) on the Canary island La Palma with a 97-cm aperture (Scharmer et al. 2003; Hammerschlag et al. 2006). Special design characteristic of the SST is an entrance vacuum window in lens shape and consequently a relatively simple optical setup with a limited number of optical surfaces. As each surface adds its fabrication deviation, a limited number of surfaces favours reaching high optical quality of the system as a whole, required for high-resolution observations. Other particular characteristics are a relatively slender tower and on top the so-called Turret, which consists of the entrance window and two folding mirrors under $45^{\circ}$. This system directs the light downward. This Turret is made as compact as possible around the optical beam and possesses large bearings with incorporated gears resulting in a very high stiffness and precision. Consequently, the compact Turret minimizes the disturbance of the surrounding air and is at the same time very stable against wind shaking.

The practical limit for vacuum telescopes is probably a little over a 1-m diameter, only slightly larger than the SST. The reason is the support problem, the same as earlier encountered for stellar lens telescopes. For the latter the practical aperture limit of about $1 \mathrm{~m}$ was reached a long time ago and, consequently, a move to mirror telescopes followed. An alternative for vacuum is a telescope filled with helium instead of air. The harmful effects of heat inside the telescope are greatly reduced, although not as much as in the case of vacuum. It avoids the large forces on vacuum windows, but still the support problem remains. For future very large solar telescopes, a solution is a mirror telescope, however not in a dome with a slit, but with the primary beam as open as possible, fully exposed to the natural airflow.

\section{Open-Telescope Technology}

The Dutch Open Telescope (DOT) was the pioneering demonstrator of the open-telescope technology now pursued in the German GREGOR. The 45-cm DOT, see Fig. 1, became an outstanding supplier of solar-atmosphere movies sampling the photosphere and chromosphere simultaneously at up to 0.2 arcsec resolution in the short-wavelength part of the visible spectrum.

Mechanical and thermal ingredients for this successful construction are: (i) primary beam completely open to natural wind flushing, (ii) effective water-cooling and air-suction near the diaphragm in prime focus, (iii) thermal equilibrium of the telescope structure parts with the surrounding air using passive methods, (iv) minimum heat production by telescope parts like drive motors, power supplies for cameras and other electronics, (v) stiff design of the telescope structure and drives to prevent wind shaking, (vi) no large objects at the telescope level during observations, reached with the completely open foldable tent construction based on strongly tensioned cloth. Additional ingredients are the use of relatively simple optics and large-volume image restoration.

\section{Developments to Large Solar Telescopes}

Development directions for application of the mechanical, thermal and optical ingredients for large 3- to 5-m mirrors are: (i) Open constructions with principal stiff overall geometries and in addition carefully designed joints to maintain geometry stiffness. An extremely stiff, still relatively lightweight, wind-stable construction can be realized. The DOT possesses a 

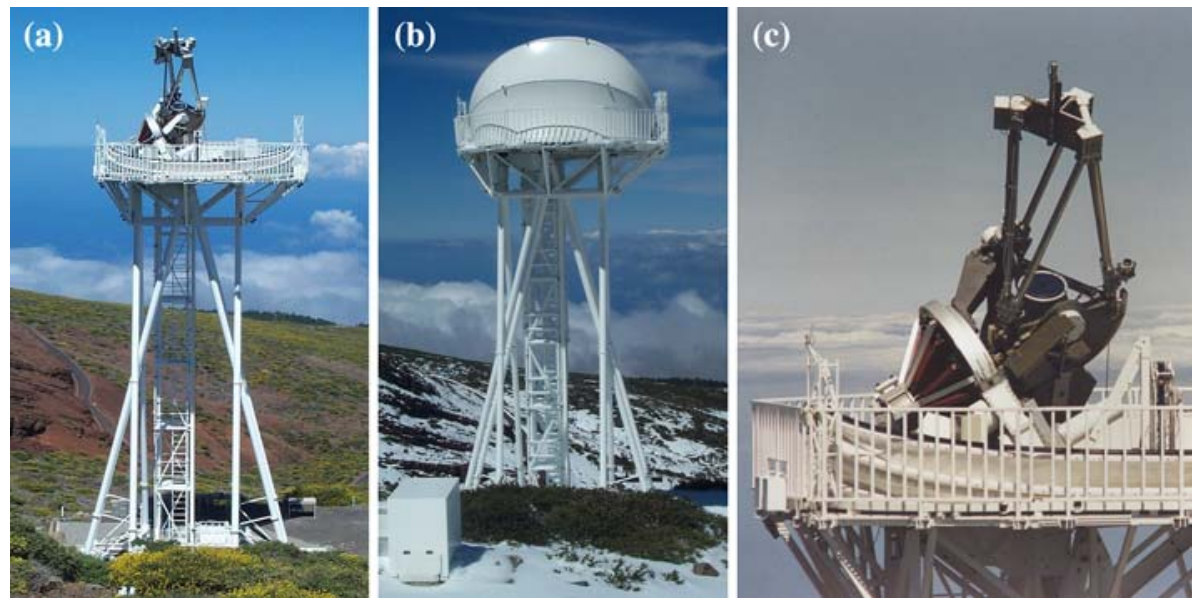

Fig. 1 The Dutch Open Telescope (DOT) with 45-cm primary mirror at 2,350 $\mathrm{m}$ altitude on La Palma. a The DOT in operation. The $15-\mathrm{m}$ tower and the telescope are sufficiently transparent to not disturb the wind, which maintains temperature homogeneity in and around the telescope. At sufficient wind strength $(7 \mathrm{~km} / \mathrm{h}$ can be enough depending on the wind direction) the larger temperature fluctuations occurring near ground level do not reach the telescope. The special tower geometry keeps the platform parallel to the ground even under strong wind loads. The clamshell canopy is opened completely for observations. b When not in operation the telescope is protected by closing the folding canopy. It is made of strong tensioned polyester cloth with an outer PVDF coating on which snow and ice do not stick. The canopy can be opened and closed within a few minutes in winds up to $100 \mathrm{~km} / \mathrm{h}$. When closed it can withstand much stronger winds and it has already survived storms of $200 \mathrm{~km} / \mathrm{h}$. c Telescope close-up. The primary mirror and the optical beam to the primary focus are fully open to wind. The DOT was the first telescope showing that such an open-air path can permit diffraction-limited resolution. Note that the primary mirror is located well above the declination axis of the equatorial mount. It sticks out high above the platform into full wind flushing

mount, which is in fact suitable for a $2.5-\mathrm{m}$ diameter mirror (Hammerschlag et al. 2007). The $45-\mathrm{cm}$ mirror was originally a first experiment in this mount. The outstanding results demonstrate that the large mount does not disturb the images when there is natural wind flushing. A large mirror would have less light absorption and consequently less heating than the large mount now without a mirror in front. (ii) Larger enclosures based on the completely open foldable tent construction. Simultaneous wind- and deformation measurements on the DOT and GREGOR enclosures will provide the knowledge for computer calculations and development of large-sized enclosures. Additional seeing measurements will provide more insight into the effects of an open, half-open or only partly-open dome on the wind turbulence and seeing.

Figure 2 shows a design for a large high-resolution solar telescope with the ingredients summed up above. The canopy leaves the telescope completely open during observations. Under telescope and canopy is an open tower. Directly under the telescope is a small optics room. From there a closed shaft goes downward to a large optical lab at the ground. In the shaft is an evacuated tube for the transport of the image and space for cables, staircase and elevator. A light beam of a diameter of a few tens of $\mathrm{cm}$ allows image transport to the ground over many tens of meters without loss of resolution and field (Hammerschlag et al. 2007). The small optical room and relatively slender shaft will not disturb significantly the open principle, as experienced with the SST. The large optical lab at the ground permits a multitude of observational instruments. 

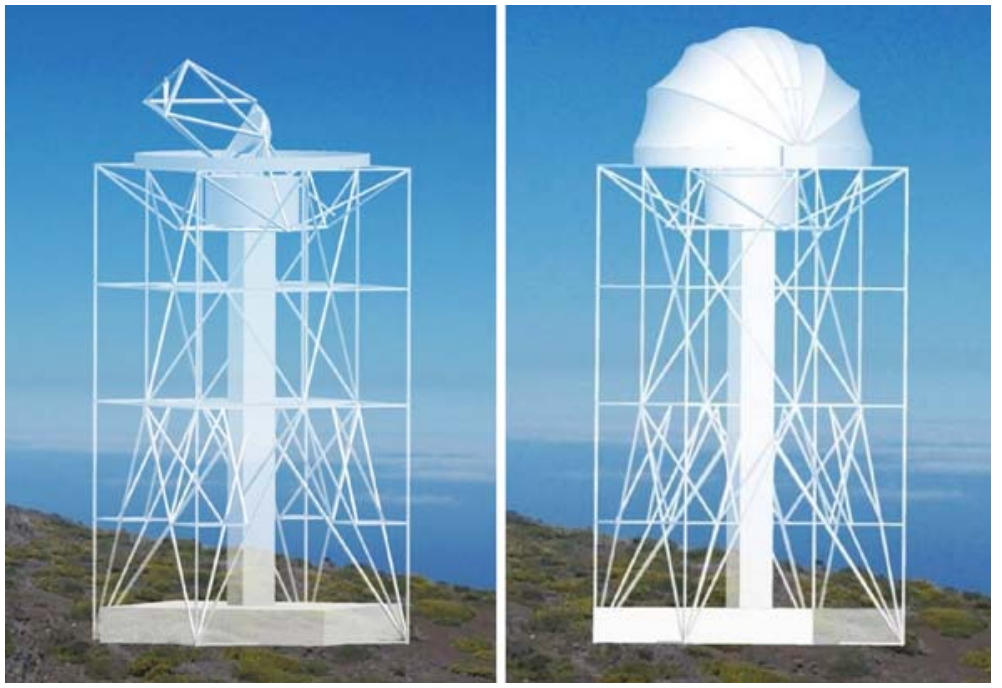

Fig. 2 Concept for a large high-resolution solar telescope. Left: in operation completely open; right: with closed canopy

Acknowledgements Further developments are supported by grants from the European Union for a design study for a European Solar Telescope (EST) and by the Dutch Technology Foundation STW for the completely open foldable tent construction, still closable in strong wind, and for seeing measurements connected to it. The SST is operated by the Royal Swedish Academy of Sciences, the DOT by Utrecht University, both at the Spanish Observatorio del Roque de los Muchachos (ORM) of the Instituto de Astrofsica de Canarias (IAC). The DOT team enjoys hospitality at the SST building.

Open Access This article is distributed under the terms of the Creative Commons Attribution Noncommercial License which permits any noncommercial use, distribution, and reproduction in any medium, provided the original author(s) and source are credited.

\section{References}

R.B. Dunn, Appl. Opt. 3, 1353 (1964)

R.B. Dunn, Sky Telescope 38(6), 368 (1969)

R.H. Hammerschlag, F.C.M. Bettonvil, A.P.L. Jägers, G.B. Scharmer, in Optomechanical Technologies for Astronomy, ed. by E. Atad-Ettedgui, J. Antebi, D. Lemke. Proceedings of the SPIE, vol. 6273, paper 623710 (2006)

R.H. Hammerschlag, F.C.M. Bettonvil, A.P.L. Jägers, R.J. Rutten, in The Physics of Chromospheric Plasmas, ed. by P. Heinzel, I. Dorotovič, R.J. Rutten. ASP Conference Series, vol. 368 (2007), pp. 573-592

G.B. Scharmer, K. Bjelksjö, T. Korhonen, B. Lindberg, B. Petterson, in Proceedings of the SPIE, vol. 4853 (2003), pp. 341-350 